\title{
Mixed Kane-Newton Multi-Body Analysis of a Dual-Arm Robotic System for On-Orbit Servicing Missions
}

\author{
Alex De Bonis ${ }^{1} \cdot$ Federica Angeletti ${ }^{2} \cdot$ Paolo lannelli $^{3} \cdot$ Paolo Gasbarri ${ }^{2} \mathbb{C}$
}

Received: 1 July 2021 / Revised: 13 July 2021 / Accepted: 22 July 2021 / Published online: 31 July 2021

(c) The Author(s) 2021

\begin{abstract}
A currently relevant topic is the development of on-orbit servicing missions designed to repair, refuel or deorbit non-cooperative spacecraft. For this purpose, it is possible to use space robotic systems composed of a main platform and one or more robotic arms. In this paper, the capacity of a dual-arm robotic system to manipulate and to deorbit a generic target will be analyzed. For this purpose, a mixed Kane-Newton multi-body model will be implemented; this model will allow to switch automatically from an open-chain configuration (target captured via a single robotic arm) to a closed-chain configuration (target captured via both robotic arms) and vice versa. The flexibility of the joints of the system and the flexibility of the components of the robotic arms will be considered in the model. The system will be properly sized to operate the deorbiting of the target. Under the hypothesis of planar motion, numerical results will be presented to validate the model and to demonstrate the correct sizing of the system.
\end{abstract}

Keywords On-orbit servicing $\cdot$ Space robotic system $\cdot$ Deorbiting $\cdot$ Multi-body model

\section{Introduction}

The presence of disused satellites and other artificial debris in orbits of scientific and commercial interest, such as LEOs and GEOs, is a more than ever relevant threat to future space missions [1]. Possible solutions to such an issue consist in capturing these orbiting objects with the scope to repair, refuel or deorbit them. Recent on-orbit servicing (OOS) and future DEBRIS REMOVAL missions currently under development confirm the growing interest in this field [2-5]. In [6], many possible technologies for active debris removal (ADR) tasks are analyzed; the most promising one involves the use of robotic space manipulators (RSM) composed of a main platform and one or more robotic arms. Literature about these issues is very broad and several multi-disciplinary aspects are

Paolo Gasbarri

paolo.gasbarri@uniroma1.it

1 Sapienza University of Rome, Piazzale Aldo Moro 5, 00185 Roma, Italy

2 School of Aerospace Engineering, Sapienza University of Rome, Via Salaria 851, 00138 Rome, Italy

3 Department of Mechanical and Aerospace Engineering, Sapienza University of Rome, Via Eudossiana 18, 00184 Roma, Italy involved. Robotic arms in space have been extensively used for assembly, inspection and maintenance of the ISS in a teleoperated way [7]. One of the missions where an autonomous system was used to capture a non-cooperative target via robotic arms is detailed in [8]. A preliminary OOS mission analysis and design is presented in [9], including the preliminary phases of far-range and close-range rendezvous with the target; robotic arms are used to capture the target and to install an apogee motor on the target with the aim of deorbiting the target. Another approach is presented in [10], in which the post-capture manipulation phase is conducted by means of two robotic arms with flexible elements in order to lead the target towards a docking interface mounted on the main platform. The methods most commonly used in literature to study such systems are the multi-body approaches of Newton and Kane $[11,12]$. In $[13,14]$, the problem of the safety of the capturephase is addressed with particular attention to contact dynamics between the end-effectors of the RSM and the target, while in [15], a method to establish the unknown inertial parameters of the target is discussed. In [16, 17], the flexibility of the joints of the RSM is modeled; the elasticity of the joints is primarily caused by the use of harmonic drives, i.e. a type of gear mechanism that is becoming increasingly popular for use in terrestrial and space robotic applications [18]. In [19], it is 
discussed how to operate the robotic arms, which captured the target, during the orbital transfer of the system.

The first goal of this work is to realize an analytical/numerical model that allows to switch automatically from an openchain configuration (target captured via a single robotic arm) to a closed-chain configuration (target captured via both robotic arms). This model allows to develop a specific manipulation strategy of the target and includes disturbing effects like flexibility of the components of the robotic arms (links flexibility), joints flexibility and gravitational effects on system components. The second goal of this work is to discuss the control of the closed-chain configuration during the orbital transfer of the coupled system (RSM plus target). The ability of the RSM to deorbit the target will be also demonstrated by assuming that an apogee motor is equipped to the main platform and by properly sizing the system to avoid an excessive elastic deformation of the links.

The paper is organized as follows: in Sect. 2, a general RSM will be defined from a kinematic and from a dynamic point of view, in Sect. 3, control strategies for the main platform and for the robotic arms will be presented, in Sect. 4, the RSM of interest and the different phases of the mission will be introduced, while in Sect. 5, numerical results, obtained through in-house codes developed in Matlab, will be showed and described.

\section{Kinematic and Dynamic Model}

In this paper, an RSM is considered as composed of a main platform and two robotic arms, each composed of three links. This implies that each robotic arm has three degrees of freedom. Figures 1 and 2 represent the system in open-chain configuration and in closed-chain configuration, respectively. Each arm component (link) is connected to the others by revolute joints. The connection between the terminal link of each arm and the target is modeled via a revolute joint with a limited rotational freedom to simulate the presence of a robotic wrist characterized by a mechanical limit [20]. An inertial reference frame $\left(X_{I}, Y_{I}\right)$ with origin in Earth's center of mass and a body reference frame $\left(X_{i}, Y_{i}\right)$ for each body of the system are considered to define the equations of motion. Links flexibility is taken into account under the hypothesis of flexural beams; the modal decomposition technique is used to define the elastic displacement $w_{i}$ of a generic point of the $i$-th link [21]. In particular:

$w_{i}\left(\xi_{i}, t\right)=\sum_{j=1}^{N_{m, i}} A_{j, i}(t) \phi_{j, i}\left(\xi_{i}\right)$,

with $\xi_{i}$ local coordinate with respect to the $i$-th body frame, $t$ time, $N_{m, i}$ number of modal shapes considered for the $i$-th link, $A_{j, i}$ and $\phi_{j, i} j$-th modal amplitude and $j$-th modal shape of the $i$-th link, respectively.

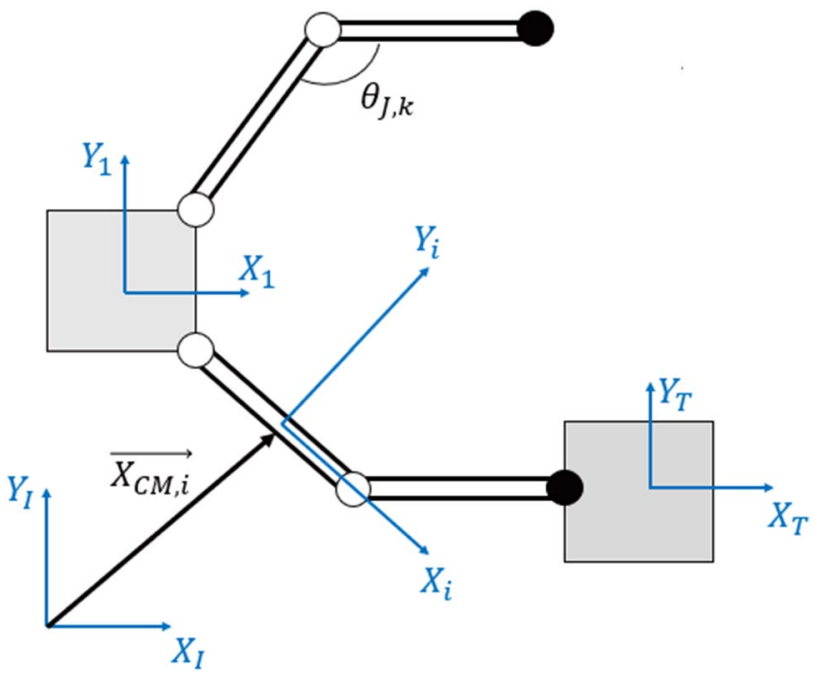

Fig. 1 Generic multi-body open-chain configuration. One arm is connected to the target while the other arm is free. The connection between first arm and target is modeled like a revolute joint. $\theta_{J, k}$ is the $\mathrm{k}$-th joint angle. It is important to underline how in this figure each arm is composed of two links while we are considering a RSM with three links for each arm

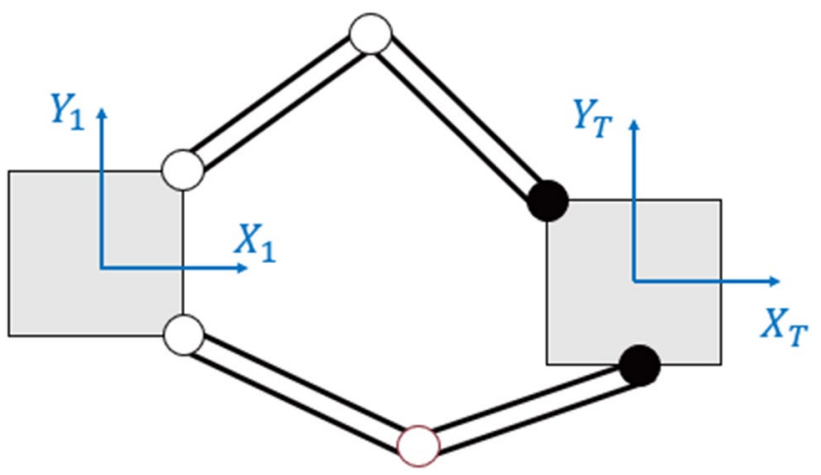

Fig. 2 Same generic multi-body closed-chain configuration. Both arms captured the target; both connection points are modeled like revolute joints

A mixed Kane-Newton formulation is implemented to define the system dynamic equations in closed-chain configuration; more details about Kane's method are reported in the Appendix of the present work. Two sets of kinematic variables are introduced to describe the state of the overall system: the first set $\underline{q}_{m}$ contains the minimum number of variables associated with the degrees of freedom of the RSM while the second set $\underline{X}_{T}$ contains the variables of the target. The set $q_{m}$, under the hypothesis of bidimensional motion, contains position and attitude angle of the main platform with respect to the inertial reference frame, joint angles of the RSM and modal amplitudes of the links while the set $\underline{X}_{T}$ contains position and attitude angle of the target with respect 
to the inertial reference frame. It is possible to define the global set of variables $\underline{Y}$ containing both $\underline{q}_{m}$ and $\underline{X}_{T}$. In detail, $\underline{Y}$ contains 24 variables in this work: 12 rigid variables (positions, attitude angles, joint angles) and 12 elastic variables (modal amplitudes) assuming to consider two modal shapes for each link of the system. The joints flexibility is modelled by coupling the $i$-th joint gear-motor with the $i$-th joint angle by means of a system consisting of a torsional spring and a damper like reported in [16-18]. For this reason, the $i$-th joint angle $\theta_{J, i}$ and the rotation angle of the output shaft of the $i$-th gear-motor $\theta_{m, i}$ become distinct variables, as observable in Fig. 3. So it is necessary to write separately the dynamic equations of RSM and the dynamic equations of joint gear-motors; in detail, the dynamic equation of the $i$-th joint gear-motor is:

$B_{\mathrm{m}, i} \ddot{\theta}_{\mathrm{m}, i}=\tau_{\mathrm{m}, i}-K_{\mathrm{m}, i}\left(\theta_{\mathrm{m}, i}-\theta_{J, i}\right)-C_{\mathrm{m}, i}\left(\dot{\theta}_{\mathrm{m}, i}-\dot{\theta}_{J, i}\right)$,

with $B_{\mathrm{m}, i}$ moment of inertia of the $i$-th motor seen from the output shaft, $\tau_{\mathrm{m}, i}$ control torque provided by the $i$-th gearmotor, $K_{\mathrm{m}, i}$ and $C_{\mathrm{m}, i}$ stiffness and damping parameters of the i-th coupling system, $\theta_{J, i} i$-th joint angle.

After writing the translational and rotational equations and the elastic equations for each body of the system, not reported here for the sake of brevity (see [21] for details), and projecting the equations of RSM into Kane's space, it is possible to write the dynamic equations of the coupled system as follows: with $J$ Jacobian matrix of the RSM, $M$ and $M_{T}$ mass matrices of the RSM and of the target, respectively, $\underline{V}$ vector containing non-linear and elastic terms of the RSM, $\underline{F}_{\text {ext }}$ vector containing external forces and torques acting on the RSM (including gravity gradient torques and gravitational forces), $\underline{V}_{T}$ and $\underline{F}_{\mathrm{ext}, T}$ the same for the target. The term $\underline{u}_{m}$ can be subdivided as follows:

$\underline{u}_{m}=\left[\begin{array}{l}\underline{u}_{\mathrm{B}} \\ \underline{u}_{\mathrm{J}} \\ \underline{u}_{\mathrm{e}}\end{array}\right]$,

with $\underline{u}_{\mathrm{B}}$ control forces on the main platform, $\underline{u}_{\mathrm{e}}$ projection of the control torques acting on robotic arms on the modal base and $\underline{u}_{\mathrm{J}}$ defined as follows:

$\underline{u}_{\mathrm{J}}=-K_{\mathrm{m}}\left(\underline{\theta}_{\mathrm{J}}-\underline{\theta}_{m}\right)-C_{m}\left(\underline{\dot{\theta}}_{J}-\underline{\dot{\theta}}_{m}\right)$

with $K_{m}$ and $C_{m}$ diagonal matrices containing stiffness and damping parameters related to joint flexibility, $\underline{\theta}_{J}$ vector containing all joint angles of the system and $\underline{\theta}_{m}$ vector containing all gear-motor angles of the system. It is important to underline that joint angles do not directly "see" the action of the control torques provided by joint motors due to joint flexibility. Finally, $\underline{R}_{\mathrm{cl}}$ is a vector containing the reaction forces between RSM and target at the connection points, while $W_{\mathrm{EE}}$ and $W_{\mathrm{T}}$ are matrices that project these reaction forces

$\left[\begin{array}{cc}J^{T} M J & 0 \\ 0 & M_{T}\end{array}\right] \ddot{\underline{Y}}=-\left[\begin{array}{cc}J^{T} M \dot{J} & 0 \\ 0 & 0\end{array}\right] \underline{\dot{Y}}+\left[\begin{array}{c}J^{T}\left(\underline{V}+\underline{F}_{\mathrm{ext}}\right) \\ \underline{V}_{T}+\underline{F}_{\mathrm{ext}, T}\end{array}\right]+\left[\begin{array}{c}\underline{u}_{m} \\ \underline{0}\end{array}\right]+\left[\begin{array}{c}J^{T} W_{\mathrm{EE}} \\ W_{T}\end{array}\right] \underline{R}_{\mathrm{cl}}$,

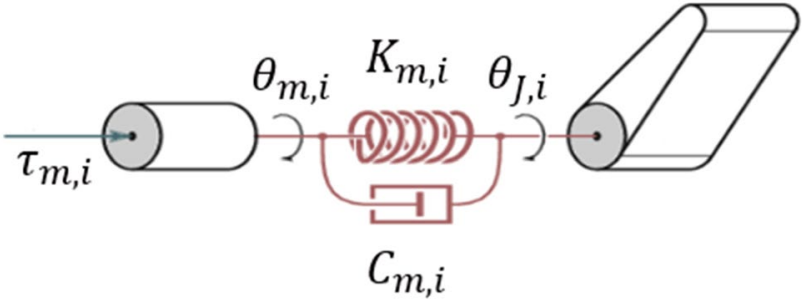

Fig. 3 Flexible joint representation into dynamic equations of RSM and target, respectively. In Fig. 4, the inertial components of these internal reaction forces acting on the system are represented; in detail, it is possible to observe that the reaction forces acting on the target is equal and opposite with respect to those acting on the last links of the RSM due to the action-reaction principle.

It is possible to rewrite (3) in the following compact way:

$$
M_{g} \underline{\ddot{Y}}=C \underline{\dot{Y}}+\underline{\mathrm{NL}}+\underline{u}+W \underline{R}_{\mathrm{cl}}
$$

Fig. 4 Reaction forces between target and terminal links of RSM. $R_{1}, R_{2}, R_{3}$ and $R_{4}$ are the inertial components of $\underline{R}_{\mathrm{cl}}$

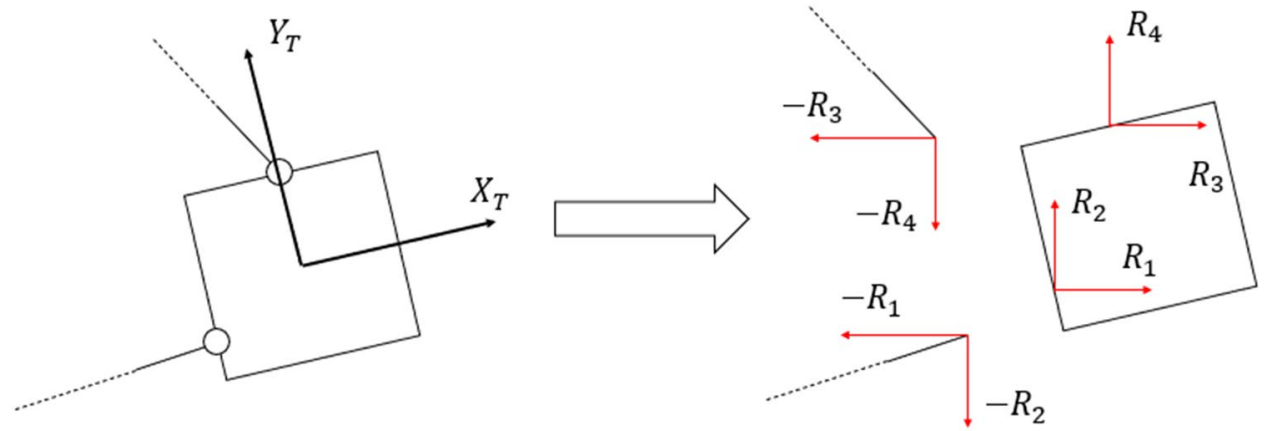


Writing (2) for each gear-motor in a compact form and considering the coupling with (6) we obtain the following system of dynamical equations:

$\left[\begin{array}{cc}M_{\mathrm{g}} & 0 \\ 0 & B_{m}\end{array}\right] \ddot{\ddot{Y}}^{*}=\left[\begin{array}{ll}C & 0 \\ 0 & 0\end{array}\right] \underline{\dot{Y}}^{*}+\left[\begin{array}{c}\mathrm{NL} \\ \underline{\tau}_{m}\end{array}\right]+\left[\begin{array}{c}\underline{u} \\ -\underline{u}_{\mathrm{J}}\end{array}\right]+\left[\begin{array}{c}W \underline{R}_{\mathrm{cl}} \\ \underline{0},\end{array}\right]$

with $B_{m}$ diagonal matrix containing the moments of inertia of the joint motors seen by the output shafts, $\tau_{m}$ vector containing the control torques provided by the gearmotors, $\underline{Y}^{*}$ vector containing both $\underline{Y}$ and $\underline{\theta}_{m}$. To define the unknown vector $\underline{R}_{\mathrm{cl}}$, it is necessary to introduce the kinematic compatibility equations; these equations ensure the connection between RSM and target [10]. It is possible to write them in algebraic form as:

$\underline{\phi}_{T}(\underline{Y})=\underline{0}$

or in differential form as:

$A \underline{\ddot{Y}}=\underline{B}$,

with the matrix $A$ and the vector $\underline{B}$ defined as follows:

$\left\{\begin{array}{l}A=\frac{\partial \underline{\phi}}{\partial \underline{Y}} \\ \underline{B}=-\frac{\partial A}{\partial t} \dot{Y}\end{array}\right.$.

Combining Eqs. (6) and (9), it is possible to obtain the vector of reaction forces:

$\underline{R}_{\mathrm{cl}}=\left[\mathrm{A} \mathrm{M}_{\mathrm{g}}^{-1} W\right]^{-1}\left[\underline{B}-A M_{\mathrm{g}}^{-1}(C \underline{\dot{Y}}+\underline{\mathrm{NL}}+\underline{u})\right]$.

Note that to avoid numerical instability problems, it is possible to apply the Baumgarte stabilization method (BSM) to the compatibility Eq. (9) replacing the vector $\underline{B}$ with the vector:

$\underline{B}^{*}=\underline{B}-2 \alpha \underline{\dot{\phi}}_{T}-\beta^{2} \underline{\phi}_{\mathrm{T}}$,

with $\alpha$ and $\beta$ real positive scalars here chosen as in [22, 23]. Supposing to have a closed-chain configuration, it is possible to simulate the release by one of two robotic arms operating on the terms $\underline{R}_{\mathrm{cl}}$ and $\underline{\phi}_{\mathrm{T}}$ with consequent passage to an openchain configuration. In closed-chain configuration, each of these terms contain 4 scalar inputs, 2 for each connection points. By setting to zero, the two terms corresponding to one of the two connection points the constraint condition disappears and the motion of the corresponding terminal link becomes independent from that of the target. In the same way, it is possible to switch from an open-chain configuration to a closed-chain configuration by imposing the presence of the two terms corresponding to one of the two connection points. This imposition is realized after the fulfillment of two "IF conditions" regarding relative position and velocity of the end-effector with respect to the designated capture point.

\section{Control Strategies}

To ensure correct pointing of the apogee motor of the main platform during the orbital transfer phase, it is necessary to develop an appropriate attitude control strategy on the main platform. The control torque $\tau_{\mathrm{B}}$ required for the attitude control of the main platform is provided by a set of momentum exchange devices. The control law here considered is a simple proportional-derivative (PD) control law:

$\tau_{\mathrm{B}}=-k_{\mathrm{p}, \mathrm{B}}\left(\theta_{\mathrm{B}}-\theta_{\mathrm{B}}^{\mathrm{des}}\right)-k_{\mathrm{d}, \mathrm{B}}\left(\dot{\theta}_{\mathrm{B}}-\dot{\theta}_{\mathrm{B}}^{\mathrm{des}}\right)$,

with $k_{\mathrm{p}, \mathrm{B}}$ and $k_{\mathrm{d}, \mathrm{B}}$ proportional and derivative control gains, $\theta_{B}$ and $\dot{\theta}_{\mathrm{B}}$ attitude angle and inertial angular velocity of the main platform respectively, $\theta_{\mathrm{B}}^{\text {des }}$ and $\dot{\theta}_{\mathrm{B}}^{\text {des }}$ desired attitude angle and angular velocity of the main platform, respectively. To control the relative position and velocity of the end-effectors with respect to the target during the capture phase, to manipulate the target and to vary the position of the system center of mass (COM) in closed-chain configuration, it is necessary to control the robotic arms by means of joint motors. It is important to remark how the control torques provided by joint motors do not act directly on the joint variables due to joint flexibility. To this aim, a Jacobian transpose control law (JTC) is used $[10,24]$. In detail:

$\underline{\tau}_{\mathrm{m}, j}=-K_{\mathrm{p}, j} J_{\mathrm{EE}, j}^{T} \underline{\mathrm{EE}, j}_{d, j}-K_{J, j}$,

with $\underline{\tau}_{m, j}$ part of $\underline{\tau}_{m}$ relative to the $j$-th arm, $\underline{\dot{\theta}}_{J, j}$ the same for $\underline{\dot{\theta}}_{J}, K_{p, j}$ and $K_{d, j}$ diagonal matrices containing proportional and derivative control gains, respectively, $\underline{e}_{\mathrm{EE}, j}$ position and attitude errors of the $j$-th end-effector with respect to desired conditions and $J_{\mathrm{EE}, \mathrm{j}}$ Jacobian matrix of the $j$-th end-effector, defined in order to relate the state of the $j$-th end-effector to the joint angles of the $j$-th robotic arm. In both cases, the desired conditions will be chosen fixed when it is necessary to keep fixed the system configuration or time-varying with polynomial law when it is necessary to reach a desired system configuration [10].

\section{System Sizing and Mission Details}

The inertial parameters of the base, the target and the links of the system are taken from [2, 25, 26]. These parameters are represented in Table 1. The 6 links ( 3 for each arm) have all the same properties; base and target have a cubic shape 
Table 1 System inertial parameters

\begin{tabular}{llll}
\hline Body & Mass $(\mathrm{Kg})$ & $J_{z, i}\left(\mathrm{Kg} \mathrm{m}^{2}\right)$ & Length $(\mathrm{m})$ \\
\hline Base & 300 & 200 & 2 \\
Target & 400 & 600 & 3 \\
Link & 36.49 & 7.23 & 1.5 \\
\hline
\end{tabular}

for which the table refers to the length of the side. While we can assume the center of mass of the base coincident
Table 2 Natural frequencies of the links

\begin{tabular}{lll}
\hline $\begin{array}{l}\text { Boundary } \\
\text { conditions }\end{array}$ & $f_{1}(\mathrm{~Hz})$ & $f_{2}(\mathrm{~Hz})$ \\
\hline PP & 30.16 & 120.64 \\
PF & 47.12 & 152.68 \\
\hline
\end{tabular}

Table 3 Base actuators parameters
Table 4 Joint actuators parameters

\begin{tabular}{ll}
\hline Parameter & Value \\
\hline$\tau_{m, i}^{\max }(\mathrm{Nm})$ & 80 \\
$B_{m, i}\left(\mathrm{Kg} \mathrm{m}^{2}\right)$ & 0.12 \\
$K_{m, i}(\mathrm{Nm} / \mathrm{rad})$ & 40000 \\
$C_{m, i}(\mathrm{Nms} / \mathrm{rad})$ & 0.002 \\
\hline
\end{tabular}

thrust $T$ and specific impulse $I_{\mathrm{sp}}$ of the apogee motor of the base are reported. In Table 4 the maximum control torque provided by each joint gear-motor $\tau_{m, i}^{\max }$ and the parameter related to joint flexibility for each joint $B_{m, i}, K_{m, i}$ and $C_{m, i}$ are represented.

\subsection{Initial Conditions and Mission Phases}

Starting from an inital condition in which the target is captured by one robotic arm and out of the operating space of the second arm, the following mission phases will be analyzed:

- target transport into the operating space of the second arm through the motion of the first arm.

- capture by the second arm.

- target manipulation and COM alignment to the thrust direction to avoid misalignment problems during the orbital transfer phase.

- orbital transfer phase.

with the geometric center of the base, it was supposed that the center of mass of the target does not coincide with the geometric center of the target. We can suppose, during the manipulation and orbital transfer phases, constant inertial parameters for each body of the system. Moreover, under the hypothesis of bidimensional motion, we can consider only the moment of inertia with respect to the out of plane axis $J_{z, i}$.

The links are characterized by a hollow square section. They are modelled as flexural beams. It is worth to note that to apply the modal decomposition technique, to describe the elastic displacement of the links, it is necessary to evaluate a proper modal base with the relevant natural frequencies in advance (see Eq. 1). For the present study, two different modal bases have been considered. One will be used to describe the dynamics of the "internal links" both in open-chain and in closed-chain configuration and one to describe the dynamics of the "external" ones in open-chain configuration. In detail, pinned-pinned (PP) end conditions are applied to the internal links, while pinned-free (PF) end conditions are applied to the external ones for the evaluation of the modal shapes. In Table 2 are reported natural frequencies of the links in both cases considering two modal shapes for each link.

The sizing of system actuators is realized on the basis of [27-29]. In Table 3 the maximum control torque provided by momentum exchange devices of the base $\tau_{B}^{\max }$,
Referring to the last phase, the COM of the system is supposed on an equatorial circular orbit of altitude $700 \mathrm{Km}$ at time zero; the goal of the orbital transfer phase is to conduct the system on an equatorial circular orbit of altitude $300 \mathrm{Km}$ to release the target causing its deorbiting due to atmospheric drag.

\section{Numerical Results}

\subsection{Transport of the Target within the Operating Space of the Second Arm}

First, it is necessary to conduct the target into the operating space of the second robotic arm starting from the initial condition represented in Fig. 5a. To carry out this manoeuvre, it is necessary to bring the end-effector of the first robotic arm towards a predefined desired position through the JTC law previously introduced. This phase is realized using a desired trajectory for the end-effector with the aim of bringing the end-effector in the desired position with zero final velocity. During this phase, the second robotic arm is also controlled with the aim of preparing it for the capture of the target. It is important to reach a rest condition at the end of this phase 
Fig. 5 Motion of the system during the first phase of manipulation: a represents the initial condition, $\mathbf{c}$ represents the final condition with the target into the operating space of the second robotic arm $\mathbf{t}=\mathbf{0 s}$

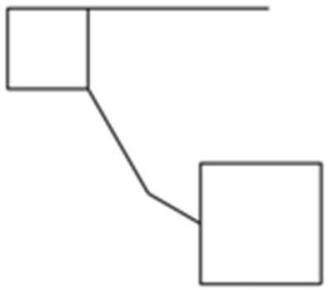

(a) $t=400 s$

$t=1200 \mathrm{~s}$

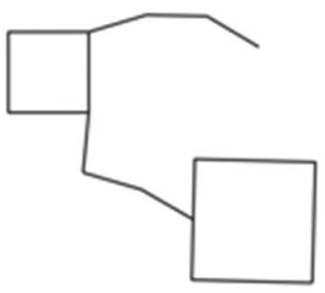

(b)

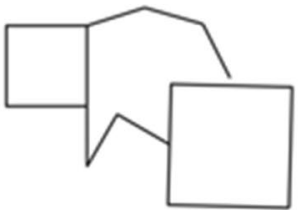

(c) before carrying out the next phase of capture in a safe mode. On account of this, it was chosen to perform this manoeuvre in ten minutes operating a subsequent maintenance phase of $10 \mathrm{~min}$.

The selection procedure of the control gains is extremely important due to the strong coupling between multi-body

(a)
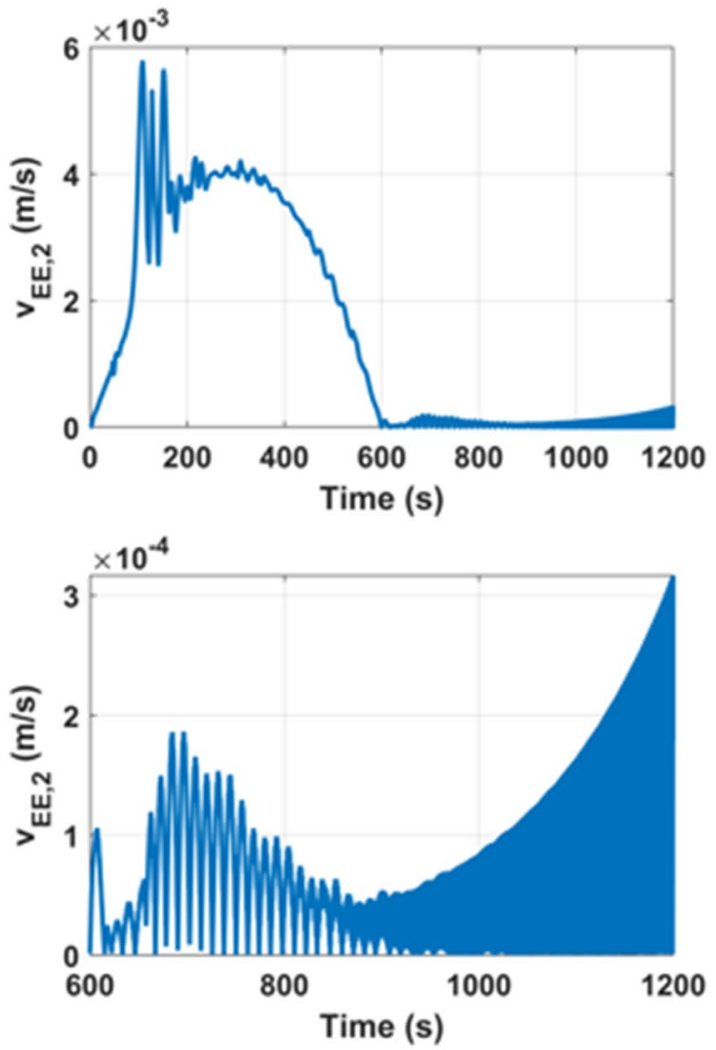

(c) dynamics, control system, links flexibility and joints flexibility. Fig. 6 represents the absolute velocity of the endeffector of the second robotic arm during this manoeuvre in two different cases. In (a) and in (c) is reported what happens when the control gains are selected, through a tradeoff analysis [30], ignoring the flexibility of the robotic
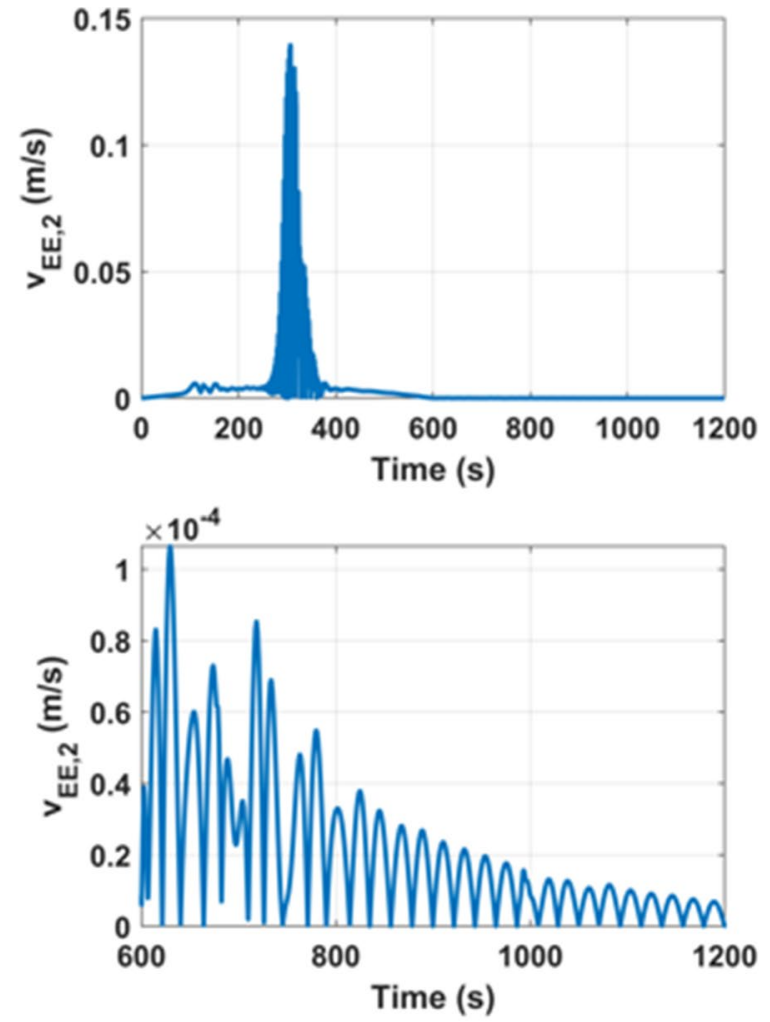

(d)

Fig. 6 Absolute velocity of the second end-effector during the first phase of manipulation. In $\mathbf{a}$ and in $\mathbf{c}$ what happens selecting the gains ignoring the elasticity of the robotic arms, in $\mathbf{b}$ and in $\mathbf{d}$ what happens

modifying the gains taking into account this aspect. In $\mathbf{a}$ and in $\mathbf{b}$ full trends in both cases, in $\mathbf{c}$ and in $\mathbf{d}$ zoom details to better understand the previous trends in both cases 
arms: during the maintenance phase this variable starts to diverge preventing the achievement of a rest condition. In (b) and in (d) is represented what happens when the selection of the control gains is performed considering flexibile links and joints: the velocity of the second end-effector decreases during the maintenance phase so a rest condition is reached.

In Fig. 5, the motion of the system during this phase is represented (from the initial condition (a) to the final condition (c)) showing the goodness of the control law once correctly sized the control gains. Figures 7 and 8 represent the coordinates of first and second end-effector, respectively, with respect to the main platform center of mass, confirming the achievement of a queit condition after the phase of maintenance.

It is interesting to observe the behavior of the attitude angle of the target in Fig. 9: the target reverses its direction of rotation when the mechanical limit of the robotic wrist is reached and the attitude angle therefore remains within a certain range; this behavior is obtained thanks to the application of a Bistop function to the joint between the robotic arm and the target to limit the relative rotation between them.

\subsection{Target Capture via the Second Robotic Arm and Target Manipulation}

The goal of the second phase is to reach a suitable configuration for the orbital transfer. First, it is necessary to capture the target via the second robotic arm switching from an open-chain to a closed-chain configuration: the model presented in Sect. 2 allows to make this step from a numerical point of view. Starting from the configuration reached at the end of the first phase and assuming the end of the first
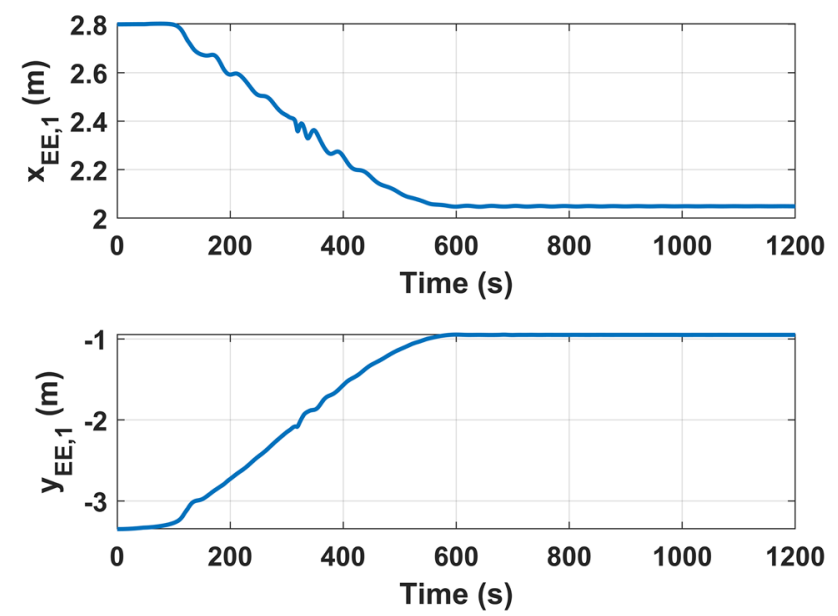

Fig. 7 Coordinates of the first end-effector with respect to the body reference frame of the main platform during the first phase of manipulation
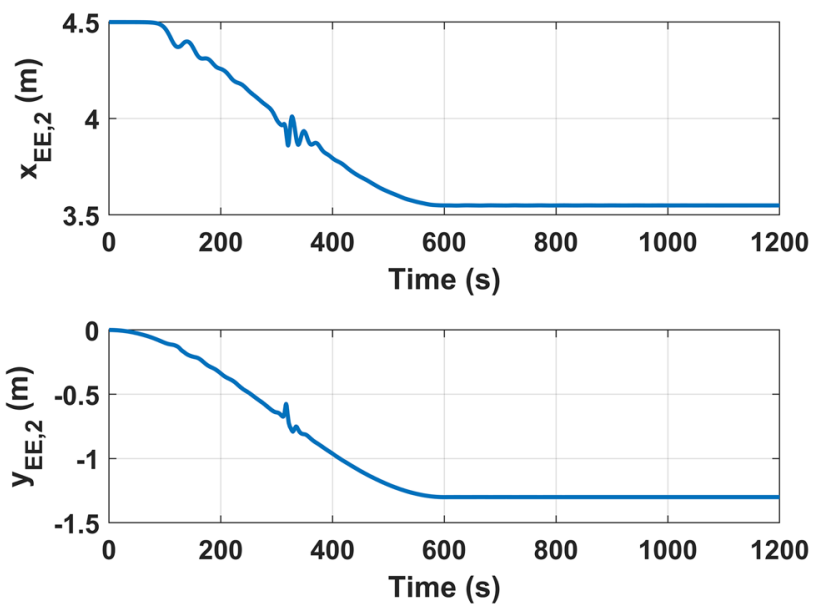

Fig. 8 Coordinates of the second end-effector with respect to the body reference frame of the main platform during the first phase of manipulation

phase as time zero, Fig. 10 represents the relative position between the second end-effector and the designated capture point of the target in terms of coordinates with respect to the main platform center of mass. After an initial approach phase, there is the capture and the relative distance between end-effector and capture point tends to zero confirming the functioning of the model and the achievement of a closedchain configuration.

Figure 11 represents the entire second manipulation phase: after the capture in (a), there is a repositioning of the target with respect to the main platform via both robotic arms in (b); then the first arm is detached and repositioned with respect to the target using the previous model to switch again from a closed-chain configuration to an open-chain configuration in (c) and finally there is the new capture by

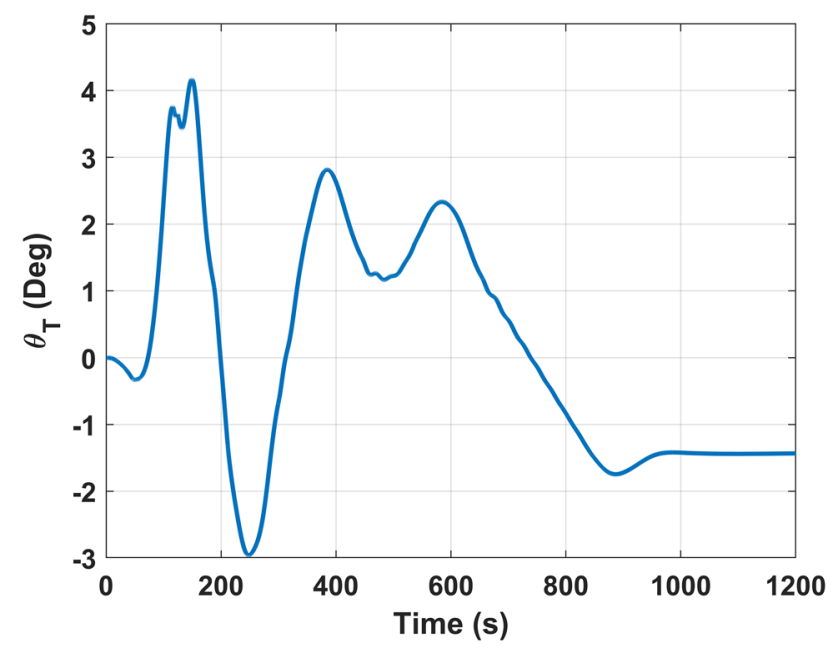

Fig. 9 Attitude angle of the target during the first phase of manipulation 

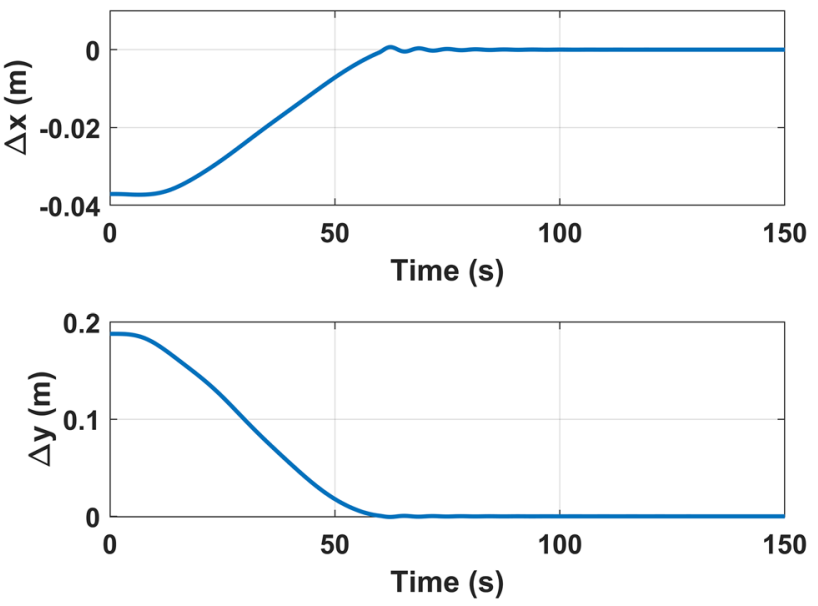

Fig. 10 Relative distance between second end-effector and designated capture point of the target in terms of coordinates with respect to the main platform center of mass during the second phase of manipulation

the first robotic arm in a new designated capture point of the target in (d).

\subsection{COM Alignment and Target Deorbiting}

Before performing the transfer orbit, it is necessary to align the center of mass of the system to the thrust direction to avoid misalignment problems: if this is not done, by igniting the motor, the system starts to rotate and we should counteract this behavior via the attitude control system. If the attitude control system saturates, there is the loss of the desired thrust direction. In fact, the apogee motor mounted on the main platform has got a fixed thrust direction, so it is necessary to move the robotic arms to align the center of mass of the system to the thrust direction with an iterative procedure: assuming the end of the second phase as time zero, Fig. 12 shows the time history of the center of mass of the system in terms of coordinates with respect to the main platform center of mass during the alignment phase. The coordinate along $y$-direction tends to zero confirming the alignment in less than $200 \mathrm{~s}$.

Fig. 11 Motion of the system during the second phase of manipulation. There are several shifts from open-chain configuration to closed-chain configuration and vice versa to reach the desired final configuration. The end of the previous phase was assumed as new zero time

$$
t=150 s
$$

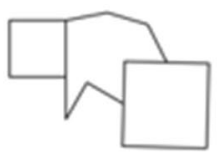

(a)
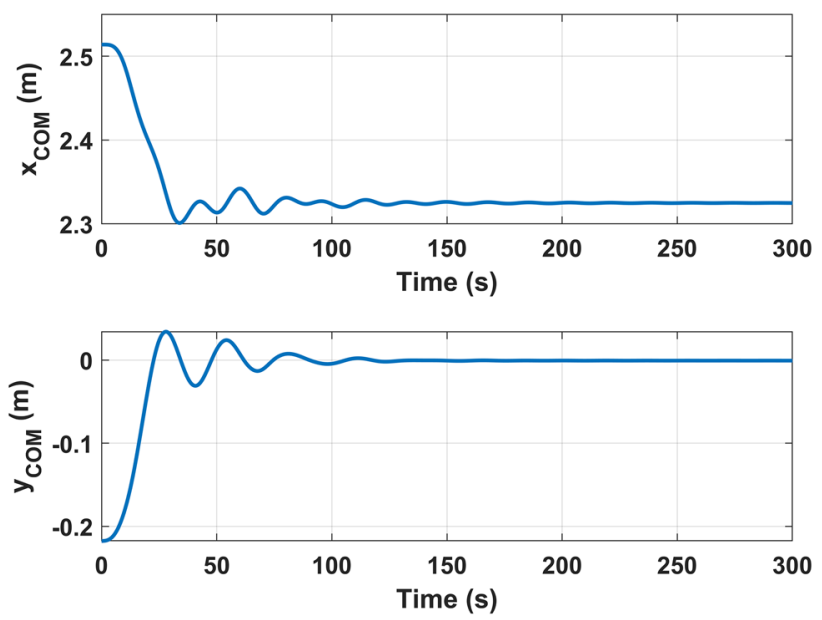

Fig. 12 Coordinates of the center of mass of the system with respect to the center of mass of the main platform

Let us now consider the last phase of the manoeuvre. It is possible to perform an Hohmann transfer orbit divided into two phases: an elliptic phase plus a circularization phase. This work focuses on the analisys of what happens to the entire system configuration during these phases and to verify the correctly sizing of the system actuators with the aim to avoid manoeuvre problems or an excessive elastic deformation of the links. Ideally the configuration should be maintained frozen during thrust intervals to avoid the impact between the main platform and the target and to mantain the alignment between COM and thrust direction previously reached; this is the approach that is followed in this paper. Fig. 13 represents the second and third joint angles of the first arm during the transfer orbit. It was chosen to refer only to these variables for the sake of brevity; all other variables have a similar behavior. The perturbances that are observable in Fig. 13 are due to the thrust (first perturbance corresponds to first thrust interval, second perturbance to second thrust interval) and imply a little variation of configuration when the thrust is turned on: joint motors try to counteract the action of the thrust to avoid relative motion between main platform and target but saturate as observable in Fig. 14. At the same time, to avoid this problem, joint motors should be $t=400 s$

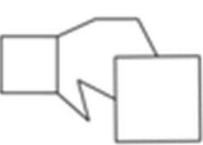

(b) $t=600 s$

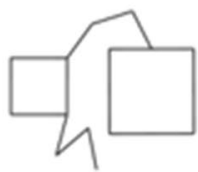

(c) $t=1200 s$

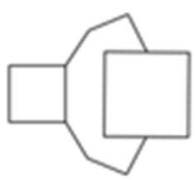

(d) 

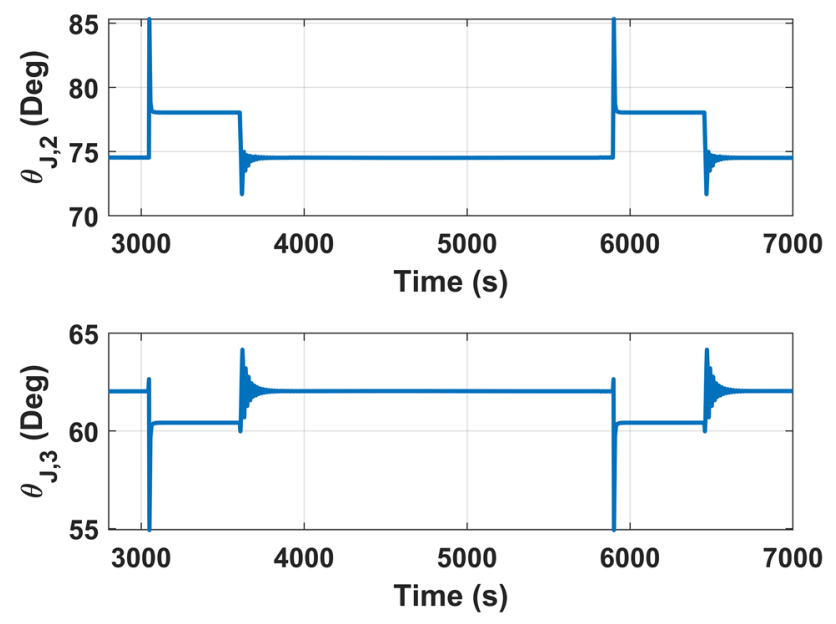

Fig. 13 Second and third joint angles of the first robotic arm during the orbital transfer phase
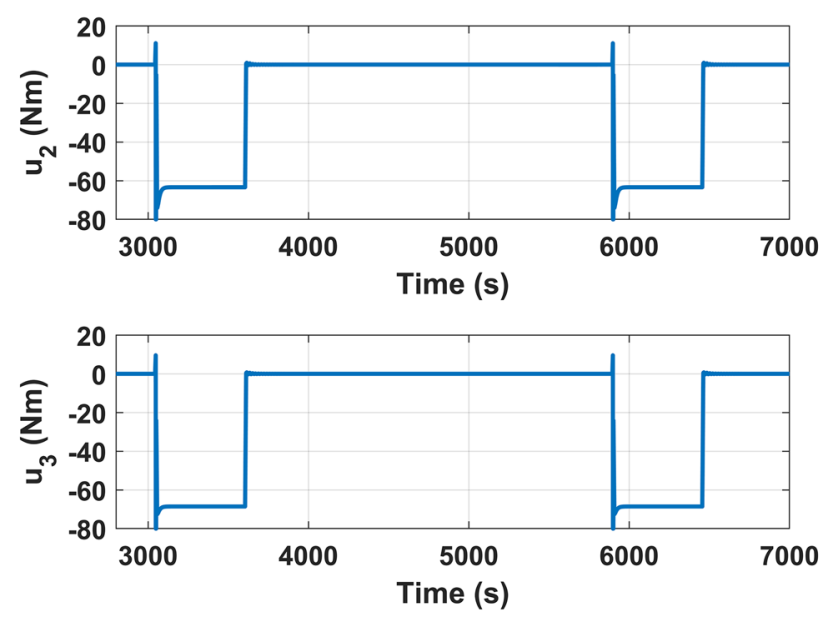

Fig. 14 Control torques provided by second and third gear-motors of the first robotic arm during the orbital transfer phase

oversized but attention must be paid to links elastic deformation. Figure 15 represents the elastic deformation of the second and third links of the first robotic arm; it is interesting to observe that when the thrust is turned on, there is an instantaneous deformation of these links (almost static since the vibrations are dampened very quickly). These deformations are completely acceptable and do not compromise the integrity of the links: this is because, accepting a small configuration change, the effect of the thrust is not fully discharged on the elastic deformation of the links. Figure 16 represents the altitude of the system: the orbit reached after the manoeuvre is not perfectly circular; however, its very small eccentricity can be considered acceptable for conducting the mission succesfully. Misalignment problems due to variation in the configuration when the thrust is turned on
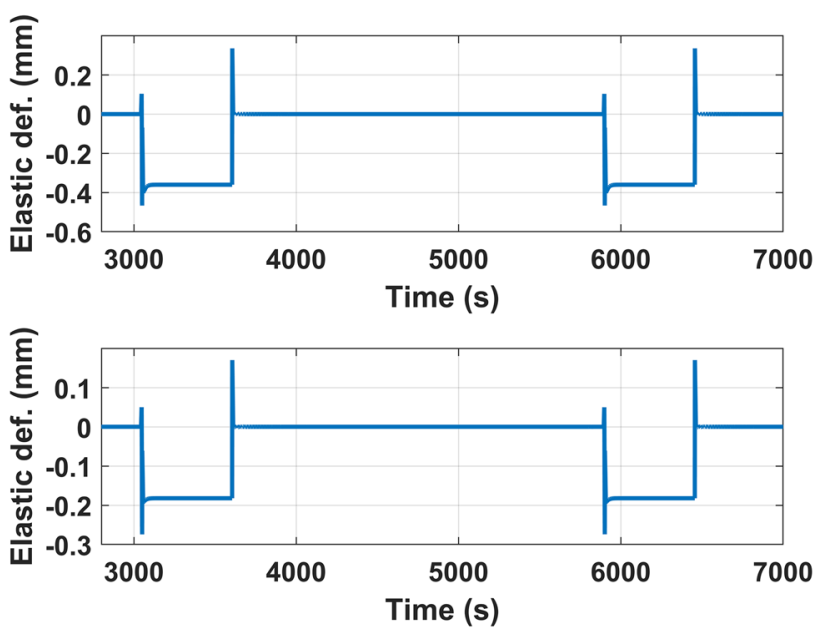

Fig. 15 Elastic deformation of second and third links of the first robotic arm during orbital transfer phase

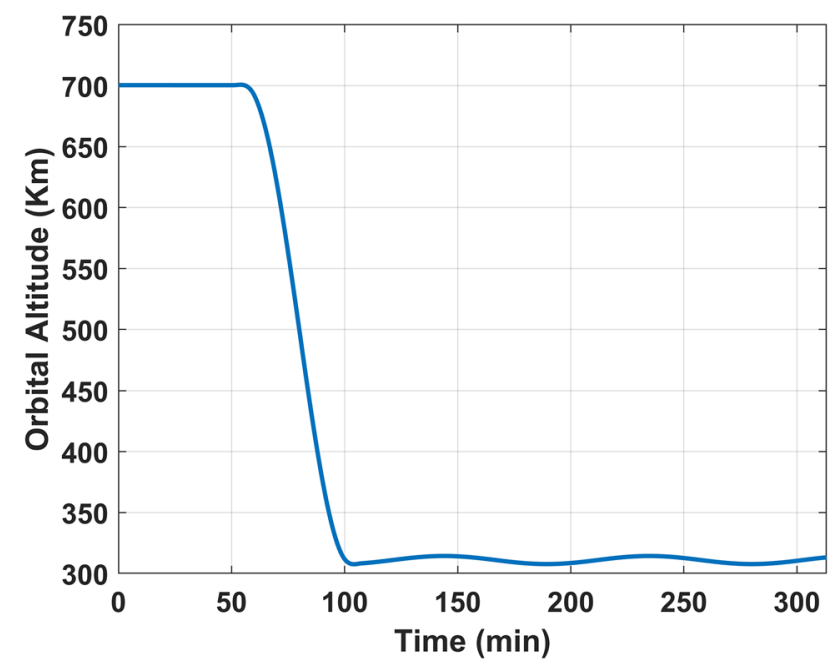

Fig. 16 Orbital altitude of the COM of the system

are limited and do not prevent to properly perform the mission. Once the target orbit is reached, the target is released by the RSM: Fig. 17 represents the trajectory of the target with respect to the RSM expressed in coordinates in the LVLH (local vertical local horizon) reference frame of the main platform. The target moves away from the RSM after the release and the RSM can be safely manoeuvred to be returned to the operational orbit.

\section{Conclusion}

This work focused on the multi-body modelling of a dualarm space flexible manipulator with the aim to demonstrate the capability of this system to capture, manipulate 


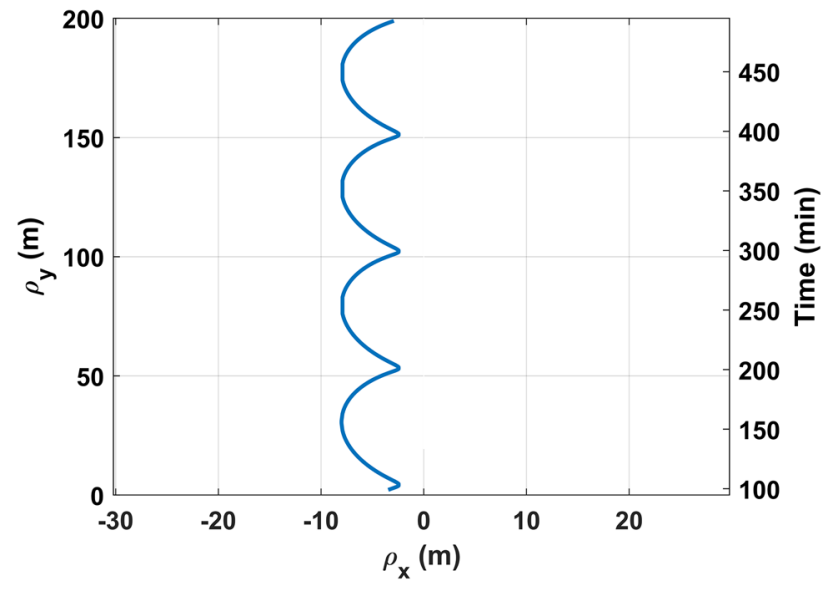

Fig. 17 Trajectory of the target with respect to the RSM after the release

and deorbit a generic target through numerical simulations. This system was composed of a main platform and two robotic arms, each with three degrees of freedom. A multibody model was developed to describe the dynamics of the system both in open-chain configuration (target captured via a single robotic arm) and in closed-chain configuration (target captured via both robotic arms). The peculiarity of the proposed model is that it allows to switch from open-chain to closed-chain configuration and viceversa using simple "IF conditions" from a numerical point of view. This model was tested developing a particular strategy of manipulation; in detail, the system was in open-chain configuration and the target was out of the operating space of the second robotic arm at time zero. This strategy allowed to reach the desired final configuration switching repeatedly from open-chain to closed-chain configuration and viceversa during the same simulation campaign. This final configuration was chosen with the aim to deorbit the target through the apogee motor of the main platform avoiding misalignment problems. Moreover, the capability of the system to deorbit the target was demonstrated: the system was sized to provide the required $\Delta V$ to lower the altitude of the target while joint motors were sized to keep the desired configuration during the transfer phase avoiding impact between the main platform and the target; also, the magnitude of the thrust has been sized to avoid excessive elastic deformation of the components of the robotic arms. Finally, it was considered flexibility of the joints of the system and it was observed that it is very important to correctly size control gains to avoid control instability due to the visco-elastic coupling between joint gear-motors variables and joint variables.

Future developments could deal with the extension of the model to the tridimensional case. It would be necessary also to carry out a robustness analysis to avoid problems due to measurement errors, communication delay and uncertainties about inertial parameters of base and target. Another possible development could be a strategy to refuel or repair the target.

\section{Appendix}

The aim of this appendix is to provide more details on the use of Kane's method for multi-body modelling of a RSM. Starting from the Newtonian state vector of the RSM $\underline{X}_{m}$ and by defining the minimum set of the Lagrangian variables $\underline{q}_{m}$ (already introduced in Sect. 2), it is possible to define the Jacobian matrix $J$ which relates the time derivative of the former to that of the latter:

$\underline{\dot{X}}_{m}=J \underline{\dot{q}}_{m}$.

Deriving Eq. (A.1) we can obtain:

$\underline{\ddot{X}}_{m}=\dot{J} \underline{\dot{q}}_{m}+J \underline{\ddot{q}}_{m}$.

Without considering the coupling with the target and the presence of control actions, the system of the Newtonian dynamics equations of the RSM can be written in the following form:

$M \underline{X}_{m}=\underline{V}+\underline{F}_{e x t}+W_{m} \underline{R}_{m}$,

with $\underline{R}_{m}$ vector containing the internal reaction forces exchanged between the links of the RSM and $W_{m}$ matrix that projects such reaction forces into the dynamics equations. Combining the Eqs. (A.2) and (A.3) and premultiplying the left and right ends of Eq. (A.3) by the term $J^{T}$, we obtain the dynamics equations of the RSM as follows:

$J^{T} M J \ddot{q}_{m}+J^{T} M \dot{J} \underline{\dot{q}}_{m}=J^{T}\left(\underline{V}+\underline{F}_{\mathrm{ext}}\right)+J^{T} W_{m} \underline{R}_{m}$.

It is possible to demonstrate that the Jacobian matrix is the null space of the matrix $W_{m}$, for which:

$J^{T} W_{m}=0$.

On account of this, we can rewrite the (A.4) in the definitive Kane's form:

$J^{T} M J \underline{\ddot{q}}_{m}+J^{T} M \dot{j} \dot{\dot{q}}_{m}=J^{T}\left(\underline{V}+\underline{F}_{\mathrm{ext}}\right)$.

Considering the presence of control actions and using a mixed Kane-Newton approach to include the coupling between the RSM and the target, we obtain the Eq. (3) described in Sect. 3. In short, the most important advantages offered by the Kane's formulation are: 
- the use of a minimum number of variables and dynamics equations for the RSM with consequent advantage from a computational point of view.

- the absence of the internal reaction forces of the RSM in the dynamics equations with consequent absence of internal compatibility equations (which would be necessary to determine such unknown reaction forces).

Funding Open access funding provided by Università degli Studi di Roma La Sapienza within the CRUI-CARE Agreement.

\section{Declarations}

Conflict of interest The authors declare that they have no conflict of interest.

Open Access This article is licensed under a Creative Commons Attribution 4.0 International License, which permits use, sharing, adaptation, distribution and reproduction in any medium or format, as long as you give appropriate credit to the original author(s) and the source, provide a link to the Creative Commons licence, and indicate if changes were made. The images or other third party material in this article are included in the article's Creative Commons licence, unless indicated otherwise in a credit line to the material. If material is not included in the article's Creative Commons licence and your intended use is not permitted by statutory regulation or exceeds the permitted use, you will need to obtain permission directly from the copyright holder. To view a copy of this licence, visit http://creativecommons.org/licenses/by/4.0/.

\section{References}

1. ESA, The current state of space debris, http://www.esa.int (12/10/2020). Accessed 20 Jun 2021

2. eoPortal, MEV-1 (Mission Extension Vehicle-1) and MEV-2, http://eoportal.org. Accessed 20 Jun 2021

3. eoPortal, ClearSpace-1 debris removal mission, http://eoportal. org. Accessed 20 Jun 2021

4. Saplan, A.: Robotic Servicing of Geosynchronous Satellites (RSGS), https://www.darpa.mil. Accessed on 20 Jun 2021

5. Medina, A., Tomassini, A.A., Suatoni, M., et al.: Towards a standardized grasping and refuelling on-orbit servicing for geo spacecraft. Acta. Astronaut. 134, 1-10 (2017). https://doi.org/10.1016/j. actaastro.2017.01.022

6. Mark, C.P., Kamath, S.: Review of active space debris removal methods. Space Policy 47, 194-206 (2019). https://doi.org/10. 1016/j.spacepol.2018.12.005

7. Caron, M., Mills, I.: Planning and Execution of Tele-Robotic Maintenance Operations on the ISS. SpaceOps 2012 Conference 11 June 2012-15 June 2012, Stockholm(Sweden), http://arc.aiaa. org https://doi.org/10.2514/6.2012-127263.

8. eoPortal, STP-1 (Space Test Program-1)/Orbital Express, http:// eoportal.org. Accessed on 20 Jun 2021

9. Castronuovo, M.M.: Active space debris removal—a preliminary mission analysis and design. Acta Astronaut. 69, 848-859 (2011). https://doi.org/10.1016/j.actaastro.2011.04.017
10. Stolfi, A., Gasbarri, P., Misra, A.K.: A two-arm flexible space manipulator system for post-grasping manipulation operations of a passive target object. Acta Astronaut. 175, 66-78 (2020). https:// doi.org/10.1016/j.actaastro.2020.04.045

11. Høifødt, H.: Dynamic modeling and simulation of robot manipulators: the Newton-Euler formulation, Master of Science in Engineering Cybernetics, Norwegian University of Science and Technology

12. Vlase, S., Negrean, I., Marin, M., Năstac, S.: Kane's methodbased simulation and modeling robots with elastic elements using finite element method. Mathematics 8(5), 805 (2020). https://doi. org/10.3390/math8050805

13. Li, S., She, Y.: Recent advances in contact dynamics and postcapture control for combined spacecraft. Progr. Aerospace Sci. 120 (2021), https://doi.org/10.13140/RG.2.2.22188.28807

14. Huang, P., Xu, Y., Liang, B.: Contact and impact dynamics of space manipulator and free-flying target. In: IEEE/RSJ International Conference on Intelligent Robots and Systems 1181-1186, (2005). https://doi.org/10.1109/IROS.2005.1545260

15. Ge, D., Wang, D., Zou, Y., Shi, J.: Motion and inertial parameter estimation of non-cooperative target on orbit using stereo vision. Adv. Space Res. 66, 1475-1484 (2020). https://doi.org/10.1016/j. asr.2020.05.029

16. De Luca, A.: A review on the control of flexible joint manipulators. IROS 2018 Workshop on Soft Robotic Modeling and Control: Bringing Together Articulated Soft Robots and Soft-Bodied Robots 5 October 2018, Madrid (Spain)

17. De Luca, A., Farina, R., Lucibello, P.: On the Control of Robots with Visco-Elastic Joints. In: Proceedings of the 2005 IEEE International Conference on Robotics and Automation April 2005, Barcelona (Spain)

18. Ulrich, S., Sasiadek, J.Z.: Methods of Trajectory Tracking for Flexible Joint Space Manipulators. In: Proceedings of the 18th World Congress, 28 August 2011-2 September 2011, Milano (Italy)

19. Felicetti, L., Gasbarri, P., Pisculli, A., Sabatini, M., Palmerini, G.B.: Design of robotic manipulators for orbit removal of spent launchers' stages. Acta Astronaut. 119, 118-130 (2016). https:// doi.org/10.1016/j.actaastro.2015.11.012

20. Functionbay, BISTOP, https://functionbay.com. Accessed on 20 Jun 2021

21. Gasbarri, P., Monti, R., Sabatini, M.: Very large space structures: non-linear control and robustness to structural uncertainties. Acta Astronaut. 93, 252-265 (2014). https://doi.org/10.1016/j.actaa stro.2013.07.022

22. Flores, P., Machado, M., Silva, M.T., Seabra, E.A.R.: A parametric study on the baumgarte stabilization method for forward dynamics of constrained multibody systems. J. Comput. Nonlinear Dynam. 6, (2011). https://doi.org/10.1115/1.4002338

23. Flores, P., Pereira, R.M.S., Machado, M., Seabra, E.A.R.: Investigation on the Baumgarte stabilization method for dynamic analysis of constrained multibody systems. In: Proceedings of EUCOMES 08 (ed. M. Ceccarelli.), https://doi.org/10.1007/9781-4020-8915-2 37

24. Buss, S.R.: Introduction to Inverse Kinematics with Jacobian Transpose, Pseudoinverse and Damped Least Squares methods. In: Proceedings of Buss2004IntroductionTI (2004)

25. Easdown, W., Felicetti, L.: A mission architecture and systems level design of navigation. Robotics and grappling hardware for an on-orbit servicing spacecraft, UKSEDS Student Space Symposium (2020)

26. Beyer, A., Grunwald, G., Heumos, M., Schedl, M., et al.: CAESAR: Space Robotics Technology for Assembly, Maintenance, 
and Repair. In: 69th International Astronautical Congress (IAC) 1-5 October 2018, Bremen (Germany)

27. Guay, P., et al., Control Moment GYRO CMG 15-45 S: a compact CMG product for agile satellites in the one ton class. In: Proceedings of the 10th European Space Mechanisms and Tribology Symposium 24-26 September 2003, San Sebastián (Spain)

28. Engineering Data CPL-2A Component Sets, www.harmonicdrive. de/3030. Accessed 20 Jun 2021

29. Bipropellant Thruster for attitude, orbit control and re-entry manoeuvres of heavy man-rated spacecraft. https://www.spacepropulsion.com. Accessed on 20 Jun 2021
30. Stolfi, A., Gasbarri, P., Sabatini, M.: A parametric analysis of a controlled deployable space manipulator for capturing a noncooperative flexible satellite. Acta Astronaut. 148, 66-78 (2018). https://doi.org/10.1016/j.actaastro.2018.04.028

Publisher's Note Springer Nature remains neutral with regard to jurisdictional claims in published maps and institutional affiliations. 